\title{
Vitamin D, diet, and lifestyle in a chronic SCI population
}

\author{
Nicholas E. Koutrakis ${ }^{1} \cdot$ Rebekah L. Goldstein $^{1} \cdot$ Palak Walia $^{1} \cdot$ Madeline M. Polak $^{1} \cdot$ Antonio A. Lazzari $^{2}$. \\ Carlos G. Tun ${ }^{3} \cdot$ Jaime E. Hart ${ }^{4,5,6} \cdot$ Eric Garshick ${ }^{5,6,7}$
}

Received: 4 October 2017 / Revised: 24 March 2018 / Accepted: 29 March 2018 / Published online: 8 August 2018

(C) International Spinal Cord Society 2018

\begin{abstract}
Study design Cross-sectional study.

Objectives Determine dietary, lifestyle, and clinical factors associated with plasma 25-hydroxyvitamin D [25(OH)D] levels in persons with chronic spinal cord injury (SCI).

Setting Veterans Affairs Medical Center in Boston, MA.

Methods 174 participants completed food frequency and health questionnaires, provided a blood sample, and underwent dual x-ray absorptiometry (DXA) to assess \%total body fat. Linear regression models were used to assess cross-sectional associations of personal, lifestyle, and nutritional factors with plasma 25(OH)D.

Results Independent factors positively associated with higher plasma 25(OH)D included vitamin D intake, age, hours of planned exercise, female sex, white race, wine consumption, and if a never or former smoker. The most important predictor of $25(\mathrm{OH}) \mathrm{D}$ was supplement intake. The majority of subjects ( $98.9 \%$ for vitamin $\mathrm{D}$ and $74.1 \%$ for calcium) did not meet the recommended daily allowance for adults from their diet alone. Level and completeness of SCI, injury duration, mobility, \% total body fat, time outside, and comorbid diseases were not associated with plasma $25(\mathrm{OH}) \mathrm{D}$.

Conclusions Plasma 25(OH)D level in chronic SCI is not associated with clinical factors specific to SCI such as injury level and completeness, injury duration, and mobility mode, but related to supplement intake and other lifestyle factors.
\end{abstract}

\section{Introduction}

Eric Garshick

eric.garshick@va.gov

1 Research and Development Service, VA Boston Healthcare System, Boston, MA, USA

2 Divison of Primary Care and Rheumatology Section, VA Boston Healthcare System, Boston University School of Medicine, Boston, MA, USA

3 Department of Physical Medicine and Rehabilitation, VA Boston Healthcare System, Boston, MA, USA

4 Department of Environmental Health, Harvard T.H. Chan School of Public Health, Boston, MA, USA

5 Channing Division of Network Medicine, Department of Medicine, Brigham and Women's Hospital, Boston, MA, USA

6 Harvard Medical School, Boston, MA, USA

7 Pulmonary, Allergy, Sleep, and Critical Care Medicine Section, Medical Service, VA Boston Healthcare System, Boston, MA, USA
Vitamin D has been linked to positive effects on bone health, cardiovascular health, and pulmonary health, among other health benefits [1-7]. Vitamin D exists in two physiologically significant forms, $\mathrm{D}_{3}$ (cholecalciferol) and $\mathrm{D}_{2}$ (ergocalciferol). Less is known, however, about the determinants of vitamin D levels in humans beyond sun exposure, from which vitamin $D_{3}$ is synthesized [1]. Studies conducted in populations without spinal cord injury (SCI) suggest that a number of lifestyle and personal factors may positively influence vitamin D levels, including greater physical activity, never-smoking status, and normal weight $[1,8-13]$.

The SCI population, in particular, is known to be at risk to have lower vitamin D levels [7, 14-18]. Due to muscular weakness and paralysis that varies based on the severity of injury, persons with SCI are predisposed to a sedentary lifestyle. It is unclear, however, whether level and severity of SCI is associated with vitamin D status or if other factors related to diet or lifestyle are important. In addition, the importance of dietary intake and reliance on vitamin $\mathrm{D}$ supplements has not been examined in this group. Vitamin 
D sufficiency can be assessed by measuring plasma 25hydroxyvitamin $\mathrm{D}[25(\mathrm{OH}) \mathrm{D}]$, which has been found to be a strong indicator of physiologically important vitamin D levels [1]. In this report, we assess the associations of a variety of clinical, dietary, and lifestyle factors with plasma 25(OH)D levels in persons participating in an SCI-health study.

\section{Methods}

\section{Subjects}

Between 5/31/2013 and 8/8/2016, 183 participants with chronic SCI taking part in a study to assess factors associated with respiratory and bone health at VA Boston were asked to complete a food frequency and dietary supplement questionnaire. Participants were recruited from persons receiving care at VA Boston, by direct mail to persons who had received care at Spaulding Rehabilitation Hospital, members of the National Spinal Cord Injury Association, subscribers to New Mobility Magazine, and from the greater Boston area through advertisement. Persons were eligible if they were 22 years of age or older, one or more years after injury, were not ventilator-dependent, did not have a tracheostomy, and had no other neuromuscular disease. The Institutional Review Boards at VA Boston Healthcare System and Harvard Medical School approved the protocol and informed consent was obtained.

\section{Food frequency and health questionnaires}

A validated semi-quantitative food frequency questionnaire (FFQ) [19] developed at the Harvard University T.H. Chan School of Public Health [20-22] was mailed out to participants before their scheduled study visit to complete on their own. This standardized questionnaire asked the subject to report on the current use of vitamins, including vitamin D and calcium, and to recall average dietary intake of a wide range of foods and beverages over the past year by answering a series of questions about various serving sizes. The answers to the items on the questionnaire are converted to daily intake of various nutrients and partitions total vitamin $\mathrm{D}$ intake into supplements and dietary intake by computerized processing at the Harvard T.H. Chan School of Public Health Nutrition Department (https://regepi.bwh. harvard.edu/health/nutrition.html). Participants completed the FFQ before the visit on a separate day and brought it in with them. If the participant left the questionnaire at home or forgot to fill it out, an additional FFQ was provided if necessary and was mailed back in shortly after the visit.

A health questionnaire based on the American Thoracic Society Questionnaire [23] with additional questions was used to obtain a standardized history that included personal characteristics. These included tobacco smoking, beer, wine, and liquor consumption in the past year (frequency and quantity), usual mobility mode (motorized wheelchair, wheelchair, use of cane/walker, or ability to walk unassisted more than half the time), and physician-diagnosed diseases such as asthma, chronic obstructive pulmonary disease (COPD), emphysema or chronic bronchitis, heart disease treated in the last 10 years, diabetes, and hypertension [24]. Persons were also asked how much time they spent in a typical week doing a planned exercise program and then asked to list the activity and how many minutes per week they participated. Examples of planned exercise included physical therapy, stretching and range of motion exercises, weight training, swimming, wheeling, walking, running, and sports. A sun exposure questionnaire based on recommendations from McCarty (2008) [25] was used to evaluate how many hours participants spent outdoors in a typical weekday and weekend day over the past month and how often they used sunscreen. Respondents were asked to report time spent outside between sunrise and sunset on a typical weekday and on a typical weekend day in the past month (no time outside, <15 min/day, 15-30 min/day, $>30$ $\min -1 \mathrm{~h} /$ day, $>1-2$ h/day, $>2-4$ h/day, $>4-6 \mathrm{~h} /$ day, $>6-8 \mathrm{~h} /$ day, $>8 \mathrm{~h} /$ day) and sunscreen use (usually $\geq 50 \%$, sometimes, and never).

\section{Neurological exam, stature, and weight}

Motor level and completeness of injury were assessed based on the American Spinal Injury Association Impairment Scale (AIS) by record review and by exam [26, 27]. Motor incomplete SCI included AIS C (most key muscles below the neurological level grade $<3 / 5$ ) or AIS D (most muscles below the neurological level grade $\geq 3 / 5$ ). For analysis, participants were further grouped into cervical motor complete (AIS A or B) and cervical AIS C, others with motor complete (AIS A or B) and AIS C, and all others AIS D. Height was obtained by measuring the body length from top of the head to the heel of the subject in a spine position. If required, wheelchairs were weighed with and without the participant, and weight was subtracted to determine the participant's weight. Self-reported height and weight were used if severe contracture or bracing hindered measurement $(n=2)$. Body mass index was calculated from height and weight.

\section{Body composition analysis}

We used a 5th generation GE Healthcare iDXA scanner to assess total body composition, including total \%body fat, using Encore 13.60 software. Scans were performed utilizing the same densitometry technician and analyzed by a 
Table 1 Characteristics of the population $(n=174)$

\begin{tabular}{|c|c|c|c|c|}
\hline & \multicolumn{3}{|l|}{ Plasma 25(OH)D levels } & \multirow{2}{*}{$\begin{array}{l}\text { Total }(n) \text { or mean } \pm \\
\text { SD or median }(25 \text { th } \\
\text { percentile, } 75 \text { th } \\
\text { percentile) }\end{array}$} \\
\hline & Sufficient $(n)[\geq 30 \mathrm{ng} / \mathrm{mL}]$ & $\begin{array}{l}\text { Insufficient }(n) \\
{[30>n \geq 20 \mathrm{ng} / \mathrm{mL}]}\end{array}$ & Deficient $(n)[<20 \mathrm{ng} / \mathrm{mL}]$ & \\
\hline$n$ & $93(53.4 \%)$ & $56(32.2 \%)$ & $25(14.4 \%)$ & 174 \\
\hline \multicolumn{5}{|l|}{ Personal } \\
\hline \multicolumn{5}{|l|}{ Sex } \\
\hline Male & $70(40.2 \%)$ & $49(28.2 \%)$ & $22(12.6 \%)$ & $141(81.0 \%)$ \\
\hline Female & $23(13.2 \%)$ & $7(4.0 \%)$ & $3(1.7 \%)$ & $33(19.0 \%)$ \\
\hline \multicolumn{5}{|l|}{ Race/ethnicity } \\
\hline White & $88(50.6 \%)$ & $45(25.9 \%)$ & $15(8.6 \%)$ & $148(85.1 \%)$ \\
\hline Other $^{\mathrm{a}}$ & $5(2.9 \%)$ & $11(6.3 \%)$ & $10(5.7 \%)$ & $26(14.9 \%)$ \\
\hline Age (years) & $58.6 \pm 11.3$ & $58.2 \pm 14.1$ & $52.8 \pm 12.0$ & $57.6 \pm 12.4$ \\
\hline $\mathrm{BMI}\left(\mathrm{kg} / \mathrm{m}^{2}\right)$ & $26.4 \pm 6.0$ & $28.2 \pm 5.9$ & $27.8 \pm 6.4$ & $27.2 \pm 6.1$ \\
\hline Obese $(\geq 30)$ & $20(11.5 \%)$ & $18(10.3 \%)$ & $7(4.0 \%)$ & $45(25.9 \%)$ \\
\hline Overweight $(25-<30)$ & $32(18.4 \%)$ & $21(12.1 \%)$ & $11(6.3 \%)$ & $64(36.8 \%)$ \\
\hline Normal/underweight $(<25)$ & $41(23.6 \%)$ & $17(9.8 \%)$ & $7(4.0 \%)$ & $65(37.4 \%)$ \\
\hline Total body fat $(\%)$ & $36.6 \pm 9.8$ & $38.5 \pm 8.6$ & $37.7 \pm 9.8$ & $37.4 \pm 9.4$ \\
\hline Education (years) & $15.4 \pm 2.7$ & $14.7 \pm 3.8$ & $13.9 \pm 2.5$ & $14.9 \pm 3.1$ \\
\hline \multicolumn{5}{|l|}{ Season of visit } \\
\hline Winter & $22(12.6 \%)$ & $12(6.9 \%)$ & $4(2.3 \%)$ & $38(21.8 \%)$ \\
\hline Spring & $19(10.9 \%)$ & $10(5.8 \%)$ & $7(4.0 \%)$ & $36(20.7 \%)$ \\
\hline Summer & $28(16.1 \%)$ & $16(9.2 \%)$ & $8(4.6 \%)$ & $52(29.9 \%)$ \\
\hline Autumn & $24(13.8 \%)$ & $18(10.3 \%)$ & $6(3.5 \%)$ & $48(27.6 \%)$ \\
\hline \multicolumn{5}{|l|}{ SCI level and completeness } \\
\hline Cervical motor complete \& AIS C & $20(11.5 \%)$ & $15(8.6 \%)$ & $5(2.9 \%)$ & $40(23.0 \%)$ \\
\hline Other motor complete \& AIS C & $38(21.8 \%)$ & $20(11.5 \%)$ & $7(4.0 \%)$ & $65(37.4 \%)$ \\
\hline All AIS D & $35(20.1 \%)$ & $21(12.1 \%)$ & $13(7.5 \%)$ & $69(39.7 \%)$ \\
\hline \multicolumn{5}{|l|}{ Mobility mode } \\
\hline Motorized wheelchair & $19(10.9 \%)$ & $10(5.8 \%)$ & $7(4.0 \%)$ & $36(20.7 \%)$ \\
\hline Hand-propelled wheelchair & $47(27.0 \%)$ & $26(14.9 \%)$ & $7(4.0 \%)$ & $80(46.0 \%)$ \\
\hline Walk with aid & $9(5.2 \%)$ & $10(5.8 \%)$ & $4(2.3 \%)$ & $23(13.2 \%)$ \\
\hline Walk without assistance & $18(10.3 \%)$ & $10(5.8 \%)$ & $7(4.0 \%)$ & $35(20.1 \%)$ \\
\hline Injury duration (years) & $22.2 \pm 14.3$ & $18.6 \pm 10.4$ & $21.2 \pm 11.9$ & $20.9 \pm 12.9$ \\
\hline \multicolumn{5}{|l|}{ Comorbid diseases } \\
\hline Asthma & $11(6.3 \%)$ & $5(2.9 \%)$ & $5(2.9 \%)$ & $21(12.1 \%)$ \\
\hline COPD & $12(6.9 \%)$ & $7(4.0 \%)$ & $1(0.6 \%)$ & $20(11.5 \%)$ \\
\hline $\mathrm{OLD}^{\mathrm{b}}$ (asthma or COPD) & $21(12.1 \%)$ & $11(6.3 \%)$ & $5(2.9 \%)$ & $37(21.3 \%)$ \\
\hline Diabetes & $17(9.8 \%)$ & $6(3.5 \%)$ & $5(2.9 \%)$ & $28(16.1 \%)$ \\
\hline Hypertension & $36(20.7 \%)$ & $18(10.3 \%)$ & $9(5.2 \%)$ & $63(36.2 \%)$ \\
\hline Heart disease, last 10 years & $16(9.2 \%)$ & $5(2.9 \%)$ & $1(0.6 \%)$ & $22(12.6 \%)$ \\
\hline \multicolumn{5}{|l|}{ Daily intake, median (25th, 75 th percentile) } \\
\hline Total vitamin D intake (IU) & $1217(655,2220)$ & $577(231,1224)$ & $260(125,367)$ & $834(327,2077)$ \\
\hline $2000-4796$ & $36(20.7 \%)$ & $8(4.6 \%)$ & $1(0.6 \%)$ & $45(25.9 \%)$ \\
\hline $1000-<2000$ & $18(10.3 \%)$ & $10(5.8 \%)$ & $2(1.2 \%)$ & $30(17.2 \%)$ \\
\hline $500-<1000$ & $24(13.8 \%)$ & $15(8.6 \%)$ & $1(0.6 \%)$ & $40(23.0 \%)$ \\
\hline$<500$ & $15(8.6 \%)$ & $23(13.2 \%)$ & $21(12.1 \%)$ & $59(33.9 \%)$ \\
\hline
\end{tabular}


Table 1 (continued)

\begin{tabular}{|c|c|c|c|c|}
\hline & \multicolumn{3}{|l|}{ Plasma 25(OH)D levels } & \multirow{2}{*}{$\begin{array}{l}\text { Total }(n) \text { or mean } \pm \\
\text { SD or median }(25 \text { th } \\
\text { percentile, } 75 \text { th } \\
\text { percentile })\end{array}$} \\
\hline & Sufficient $(n)[\geq 30 \mathrm{ng} / \mathrm{mL}]$ & $\begin{array}{l}\text { Insufficient }(n) \\
{[30>n \geq 20 \mathrm{ng} / \mathrm{mL}]}\end{array}$ & Deficient $(n)[<20 \mathrm{ng} / \mathrm{mL}]$ & \\
\hline Vitamin D intake from diet (IU) & $177(109,258)$ & $150(116,229)$ & $196(88,273)$ & $164(109,255)$ \\
\hline $300-692$ & $20(11.5 \%)$ & $5(2.9 \%)$ & $4(2.3 \%)$ & $29(16.7 \%)$ \\
\hline $200-<300$ & $20(11.5 \%)$ & $12(6.9 \%)$ & $7(4.0 \%)$ & $37(21.3 \%)$ \\
\hline $100-<200$ & $35(20.1 \%)$ & $26(14.9 \%)$ & $7(4.0 \%)$ & $68(39.1 \%)$ \\
\hline$<100$ & $20(11.5 \%)$ & $13(7.5 \%)$ & $7(4.0 \%)$ & $40(23.0 \%)$ \\
\hline Vitamin D from supplements (IU) & $1000(400,2000)$ & $400(50,1064)$ & $0(0,57)$ & $561(57,2000)$ \\
\hline $2000-4750$ & $30(17.2 \%)$ & $8(4.6 \%)$ & $1(0.6 \%)$ & $39(22.4 \%)$ \\
\hline $1000-<2000$ & $18(10.3 \%)$ & $8(4.6 \%)$ & $2(1.2 \%)$ & $28(16.1 \%)$ \\
\hline$<1000$ & $35(20.1 \%)$ & $27(15.5 \%)$ & $5(2.9 \%)$ & $67(38.5 \%)$ \\
\hline None & $10(5.8 \%)$ & $13(7.5 \%)$ & $17(19.8 \%)$ & $40(23.0 \%)$ \\
\hline Total calcium intake (mg) & $1396(959,1718)$ & $1160(633,1427)$ & $957(504,1123)$ & $1206(694,1610)$ \\
\hline $2000-3943$ & $17(9.8 \%)$ & $3(1.7 \%)$ & - & $20(11.5 \%)$ \\
\hline $1000-<2000$ & $51(29.3 \%)$ & $28(16.1 \%)$ & $12(6.9 \%)$ & $91(52.3 \%)$ \\
\hline $500-<1000$ & $17(9.8 \%)$ & $16(9.2 \%)$ & $7(4.0 \%)$ & $40(23.0 \%)$ \\
\hline$<500$ & $8(4.6 \%)$ & $9(5.2 \%)$ & $6(3.5 \%)$ & $23(13.2 \%)$ \\
\hline Calcium intake from diet (mg) & $863(573,1165)$ & $702(512,949)$ & $624(504,1057)$ & $748(534,1029)$ \\
\hline $1000-2762$ & $31(17.8 \%)$ & $10(5.8 \%)$ & $8(4.6 \%)$ & $49(28.2 \%)$ \\
\hline $750-<1000$ & $22(12.6 \%)$ & $13(7.5 \%)$ & $2(1.2 \%)$ & $37(21.3 \%)$ \\
\hline $500-<750$ & $25(14.4 \%)$ & $21(12.1 \%)$ & $9(5.2 \%)$ & $55(31.6 \%)$ \\
\hline$<500$ & $15(8.6 \%)$ & $12(6.9 \%)$ & $6(3.5 \%)$ & $33(19.0 \%)$ \\
\hline Calcium from supplements (mg) & $500(0,818)$ & $200(0,607)$ & $0(0,29)$ & $200(0,618)$ \\
\hline $1000-2220$ & $19(10.9 \%)$ & $5(2.9 \%)$ & - & $24(13.8 \%)$ \\
\hline $500-<1000$ & $29(16.7 \%)$ & $16(9.2 \%)$ & $2(1.2 \%)$ & $47(27.0 \%)$ \\
\hline$<500$ & $16(9.2 \%)$ & $12(6.9 \%)$ & $5(2.9 \%)$ & $33(19.0 \%)$ \\
\hline None & $29(16.7 \%)$ & $23(13.2 \%)$ & $18(10.3 \%)$ & $70(40.2 \%)$ \\
\hline Beer or liquor use (drinks/month) ${ }^{c}$ & $14(4,36)$ & $12(4,28)$ & $18(6,56)$ & $12(4,36)$ \\
\hline Above median $(>12)$ & $27(15.5 \%)$ & $8(4.6 \%)$ & $6(3.5 \%)$ & $41(23.6 \%)$ \\
\hline Below median $(\leq 12)$ & $27(15.5 \%)$ & $10(5.8 \%)$ & $6(3.5 \%)$ & $43(24.7 \%)$ \\
\hline None & $39(22.4 \%)$ & $38(21.8 \%)$ & $13(7.5 \%)$ & $90(51.7 \%)$ \\
\hline Wine use (drinks/month) ${ }^{\mathrm{c}}$ & $12(4,24)$ & $12(8,24)$ & $8(2,12)$ & $12(4,24)$ \\
\hline Above median $(>12)$ & $18(10.3 \%)$ & $6(3.5 \%)$ & - & $24(13.8 \%)$ \\
\hline Below median $(\leq 12)$ & $20(11.5 \%)$ & $7(4.0 \%)$ & $3(1.7 \%)$ & $30(17.2 \%)$ \\
\hline None & $55(31.6 \%)$ & $43(24.7 \%)$ & $22(12.6 \%)$ & $120(69.0 \%)$ \\
\hline \multicolumn{5}{|l|}{ Lifestyle } \\
\hline \multicolumn{5}{|l|}{ Smoking status } \\
\hline Current & $5(2.9 \%)$ & $7(4.0 \%)$ & $4(2.3 \%)$ & $16(9.2 \%)$ \\
\hline Former & $45(25.9 \%)$ & $26(14.9 \%)$ & $15(8.6 \%)$ & $86(49.4 \%)$ \\
\hline Never & $43(24.7 \%)$ & $23(13.2 \%)$ & $6(3.5 \%)$ & $72(41.4 \%)$ \\
\hline Planned exercise (h/week) & $7.2 \pm 6.1(n=59)$ & $6.0 \pm 5.3(n=36)$ & $5.0 \pm 3.5(n=12)$ & $6.5 \pm 5.6(n=107)$ \\
\hline $\begin{array}{l}\text { Stretching/range of motion (ROM)/ } \\
\text { physical therapy (h/week) }\end{array}$ & $2.7 \pm 2.7(n=24)$ & $3.5 \pm 3.7(n=17)$ & $2.3 \pm 1.4(n=6)$ & $3.0 \pm 3.0(n=47)$ \\
\hline Other exercise (h/week) & $6.8 \pm 6.1(n=53)$ & $5.0 \pm 4.4(n=31)$ & $5.1 \pm 4.0(n=9)$ & $6.0 \pm 5.4(n=93)$ \\
\hline
\end{tabular}


Table 1 (continued)

\begin{tabular}{lllll}
\hline & Plasma 25(OH)D levels & & $\begin{array}{l}\text { Total }(n) \text { or mean } \pm \\
\text { SD or median }(25 \text { th } \\
\text { percentile, } 75 \text { th } \\
\text { percentile })\end{array}$ \\
\cline { 2 - 5 } & Sufficient $(n)[\geq 30 \mathrm{ng} / \mathrm{mL}]$ & $\begin{array}{l}\text { Insufficient }(n) \\
{[30>n \geq 20 \mathrm{ng} / \mathrm{mL}]}\end{array}$ & Deficient $(n)[<20 \mathrm{ng} / \mathrm{mL}]$ & $12.9 \pm 12.4(n=163)$ \\
\hline $\begin{array}{l}\text { Time outside between sunrise and } \\
\text { sunset (h/week) }\end{array}$ & $12.2 \pm 12.1(n=87)$ & $14.6 \pm 14.6(n=52)$ & $11.9 \pm 8.0(n=24)$ & $14.4 \pm 13.0(n=97)$ \\
$\begin{array}{l}\text { Time outside between sunrise and } \\
\text { sunset, visits April through September } \\
\text { (h/week) }\end{array}$ & $14.5 \pm 13.2(n=52)$ & $15.9 \pm 14.6(n=29)$ & $11.6 \pm 8.9(n=16)$ & \\
$\begin{array}{l}\text { Sunscreen use when outdoors } \\
\quad \text { Usually ( } \geq 50 \%)\end{array}$ & $15(9.0 \%)$ & $3(1.8 \%)$ & - & $18(10.8 \%)$ \\
$\quad$ Sometimes & $13(7.8 \%)$ & $12(7.2 \%)$ & $1(0.6 \%)$ & $26(15.6 \%)$ \\
$\quad$ Never & $62(37.1 \%)$ & $40(24.0 \%)$ & $21(12.6 \%)$ & $123(73.7 \%)$ \\
\hline
\end{tabular}

${ }^{a}$ Includes 19 Black, 4 Hispanic, 2 Asian, 1 Native American

${ }^{\mathrm{b}}$ Obstructive lung disease

${ }^{c}$ Excluding non-drinkers

certified clinical densitometrist (AAL). Daily quality assurance was performed utilizing a phantom.

\section{Vitamin D analysis}

EDTA plasma was drawn and immediately delivered to the core blood research laboratory at our facility. Samples were centrifuged for $15 \mathrm{~min}$ at $2600 \mathrm{rpm}(1459 \mathrm{~g})$ at $4{ }^{\circ} \mathrm{C}$ and stored at $-80^{\circ} \mathrm{C}$. Analysis of $25(\mathrm{OH}) \mathrm{D}$ was done at the Clinical \& Epidemiologic Research Laboratory, Department of Laboratory Medicine at Children's Hospital in Boston by high performance liquid chromatography tandem mass spectrometry (HPLC-MS/MS). The assay is linear up to $100 \mathrm{ng} / \mathrm{mL}$, and sensitive to $1 \mathrm{ng} / \mathrm{mL}$. Day-to-day precision $(\% \mathrm{CV})$ at various levels of $25(\mathrm{OH}) \mathrm{D}$ ranged from 5.6 to $8.5 \%$.

\section{Analyses}

We excluded 1 person with sarcoidosis, 6 persons whose plasma $25(\mathrm{OH}) \mathrm{D}$ was unavailable, and 2 persons whose plasma 25(OH)D levels were considered to be outliers (values of 71.9 and $86.3 \mathrm{ng} / \mathrm{mL}$ ) leaving a sample size of 174 participants. General linear models (PROC GLM, SAS version 9.4) were used to assess both continuous and categorical determinants of $25(\mathrm{OH}) \mathrm{D}$. Residual plots were examined to assess goodness of fit. Variables significant at the 0.100 level in univariable models were assessed in multivariable models and adjusted for laboratory batch. A sensitivity analysis was conducted excluding 18 persons whose responses on the FFQ conflicted with their responses to supplement use on the health questionnaire. The Tukey method was used to determine the significance between multiple (>2) groups in categorical variables. Monthly alcohol intake of beer, wine, and other liquor was estimated by multiplying usual daily or weekly intake by the frequency of drinking. Since wine consumption was the only alcohol type associated with plasma $25(\mathrm{OH}) \mathrm{D}$, beer and liquor consumptions were grouped together to represent all non-wine consumption. Usual time outdoors over a week was estimated by multiplying the median of each weekday category by 5 and the median of each weekend day category by 2 (using $9 \mathrm{~h}$ for the highest category), and summing to obtain the total hours. Season was assigned based on calendar month of study visit. Trends across wine consumption categories were assessed using the median values and for smoking categories by assigning ordinal numbers. To examine the patterns of predictors with the outcome, we used the 2011 Endocrine Society's Practice guidelines (vitamin D deficiency: 25(OH)D $<20 \mathrm{ng} / \mathrm{mL}$; insufficiency: $\geq 20$ to $<30 \mathrm{ng} / \mathrm{mL}$; sufficiency: $\geq 30 \mathrm{ng} / \mathrm{mL}$ ) [28].

\section{Results}

Personal, lifestyle, and nutritional characteristics of the population are shown in Table 1. The mean (SD) age was $57.6 \pm 12.4$ years, the majority of the population was male $(81.0 \%)$ and white $(85.1 \%)$, and the mean BMI was $27.2 \pm$ $6.1 \mathrm{~kg} / \mathrm{m}^{2}$. The average injury duration of the participants was $20.9 \pm 12.9$ years and $23.0 \%$ of participants had motor complete and AIS C SCI, 37.3\% had lower levels of motor complete and AIS C SCI, and $39.7 \%$ had AIS D SCI. Most subjects used either motorized (20.7\%) or hand-propelled wheelchairs $(46.0 \%)$. Mean plasma $25(\mathrm{OH}) \mathrm{D}$ level was $31.2 \pm 10.3$ (range $10.1-56.2$ ) $\mathrm{ng} / \mathrm{mL}$. Slightly more than half of the participants were vitamin D sufficient (53.4\%), about a third were vitamin D insufficient (32.2\%), and the 
Table 2 Univariable associations with plasma vitamin D

$\beta \pm \mathrm{SE} \quad P$

Personal

Age (years)

$0.115 \pm 0.06 \quad 0.068$

BMI $\left(\mathrm{kg} / \mathrm{m}^{2}\right)$

$-0.158 \pm 0.13 \quad 0.225$

Total body fat $(\%)$

$-0.031 \pm 0.08 \quad 0.715$

Education (years)

$0.490 \pm 0.25 \quad 0.052$

Duration of injury (years)

$0.010 \pm 0.06 \quad 0.876$

Daily intake

Total vitamin D intake (per $100 \mathrm{IU}$ )

Vitamin D intake from diet (per $100 \mathrm{IU})$

$0.451 \pm 0.07 \quad<0.001$

Vitamin D from supplements (per $100 \mathrm{IU}$ )

$0.695 \pm 0.61 \quad 0.259$

$0.444 \pm 0.08<<.001$

Total calcium intake (per $100 \mathrm{mg}$ )

$0.417 \pm 0.11<0.001$

Calcium intake from diet (per $100 \mathrm{mg}$ )

$0.315 \pm 0.18 \quad 0.082$

Calcium from supplements (per $100 \mathrm{mg}$ )

$0.501 \pm 0.15$

0.001

Lifestyle

Planned exercise (h/week)

Stretching/range of motion/physical therapy (h/week)

Other exercise (h/week)

Time outside, sunrise, and sunset (h/week)

Time outside, sunrise and sunset, April-September (h/week)

$\begin{array}{ll}0.317 \pm 0.14 & 0.028 \\ -0.232 \pm 0.39 & 0.554 \\ 0.418 \pm 0.16 & 0.008 \\ -0.011 \pm 0.06 & 0.857 \\ 0.062 \pm 0.08 & 0.436\end{array}$

\begin{tabular}{|c|c|c|}
\hline & Mean $(\mathrm{ng} / \mathrm{mL}) \pm \mathrm{SE}$ & $P$ \\
\hline \multicolumn{3}{|l|}{ Personal } \\
\hline Sex & & 0.021 \\
\hline Male & $30.4 \pm 0.9$ & \\
\hline Female & $35.0 \pm 1.8$ & \\
\hline Race & & $<0.001$ \\
\hline White & $32.8 \pm 0.8$ & \\
\hline Other & $22.3 \pm 1.9$ & \\
\hline BMI & & 0.262 \\
\hline Obese $(\geq 30)$ & $29.8 \pm 1.5$ & \\
\hline Overweight $(25-<30)$ & $30.6 \pm 1.3$ & \\
\hline Normal/underweight $(<25)$ & $32.8 \pm 1.3$ & \\
\hline Season of visit & & 0.737 \\
\hline Winter & $32.5 \pm 1.7$ & \\
\hline Spring & $30.1 \pm 1.7$ & \\
\hline Summer & $30.6 \pm 1.4$ & \\
\hline Autumn & $31.7 \pm 1.5$ & \\
\hline SCI level and completeness & & 0.319 \\
\hline Motor complete cervical \& AIS C & $29.8 \pm 1.6$ & \\
\hline Other motor complete \& AIS C & $32.7 \pm 1.3$ & \\
\hline All AIS D & $30.6 \pm 1.2$ & \\
\hline Mobility mode & & 0.476 \\
\hline Motorized wheelchair & $31.1 \pm 1.7$ & \\
\hline Hand-propelled wheelchair & $32.4 \pm 1.2$ & \\
\hline Walk with aid & $29.2 \pm 2.2$ & \\
\hline Walk without assistance & $29.9 \pm 1.8$ & \\
\hline
\end{tabular}


Table 2 (continued)

\begin{tabular}{|c|c|c|}
\hline & Mean $(\mathrm{ng} / \mathrm{mL}) \pm \mathrm{SE}$ & $P$ \\
\hline \multicolumn{3}{|l|}{ Comorbid diseases } \\
\hline Asthma & $32.6 \pm 2.3$ & 0.507 \\
\hline No asthma & $31.0 \pm 0.8$ & \\
\hline COPD & $32.3 \pm 2.3$ & 0.610 \\
\hline No COPD & $31.1 \pm 0.8$ & \\
\hline $\mathrm{OLD}^{\mathrm{a}}$ (asthma or COPD) & $32.8 \pm 1.7$ & 0.295 \\
\hline No OLD & $30.8 \pm 0.9$ & \\
\hline Diabetes & $30.7 \pm 2.0$ & 0.790 \\
\hline No diabetes & $31.3 \pm 0.9$ & \\
\hline Hypertension & $32.4 \pm 1.3$ & 0.261 \\
\hline No hypertension & $30.6 \pm 1.0$ & \\
\hline Heart disease, last 10 years & $34.4 \pm 2.2$ & 0.127 \\
\hline No heart disease & $30.8 \pm 0.8$ & \\
\hline \multicolumn{3}{|l|}{ Intake } \\
\hline Beer or liquor use (drinks/month) & & 0.411 \\
\hline Above median $(>12)$ & $31.0 \pm 1.6$ & \\
\hline Below median $(\leq 12)$ & $33.0 \pm 1.6$ & \\
\hline None & $30.5 \pm 1.1$ & \\
\hline Wine use (drinks/month) & & $<0.001 P$ trend $<0.001$ \\
\hline Above median $(>12)$ & $37.0 \pm 2.0$ & \\
\hline Below median $(\leq 12)$ & $34.0 \pm 1.8$ & \\
\hline None & $29.4 \pm 0.9$ & \\
\hline \multicolumn{3}{|l|}{ Lifestyle } \\
\hline Smoking status & & $0.057 P$ trend 0.020 \\
\hline Current & $26.4 \pm 2.6$ & \\
\hline Former & $30.7 \pm 1.1$ & \\
\hline Never & $33.0 \pm 1.2$ & \\
\hline Sunscreen use when outdoors & & 0.003 \\
\hline Usually $(\geq 50 \%)^{\mathrm{b}}$ & $38.5 \pm 2.3$ & \\
\hline Sometimes & $32.6 \pm 1.9$ & \\
\hline Never & $30.2 \pm 0.9$ & \\
\hline
\end{tabular}

${ }^{\mathrm{a}}$ Obstructive lung disease

${ }^{\mathrm{b}} P=0.127$ compared to sometimes and $P=0.004$ compared to never

rest were vitamin D deficient (14.4\%). Participants consumed a median (25th, 75th percentile) $164(109,255) \mathrm{IU}$ of vitamin D daily from their diet and a median 561 $(57,2000)$ IU from supplements. All but 2 participants (98.9\%) did not meet the Institute of Medicine (IOM) recommended dietary allowance (RDA) of $600 \mathrm{IU}$ of vitamin D for adults up to 70 and $800 \mathrm{IU}$ for ages above 70 from their diet alone [29]. Similar to vitamin D, the majority of participants $(n=129,74.1 \%)$ did not reach IOM recommendations for calcium intake based on diet alone (1000 mg of calcium for ages $31-70$ and $1200 \mathrm{mg}$ for persons over the age of 70) with a median dietary calcium intake of $748(534,1029) \mathrm{mg}$. Most participants also did not drink beer or liquor $(51.7 \%)$ or wine in the past years
(69.0\%), but, of those who drank, the median (25th, 75th percentile) number of beers or drinks of liquor was 12 (4, 36) drinks per month and glasses of wine was $12(4,24)$. In addition, almost half of the participants were former smokers $(49.4 \%)$ and few were current smokers $(9.2 \%)$. Persons who spent time outdoors between sunrise and sunset spent an average of $12.9 \pm 12.5 \mathrm{~h}$ during a typical week in the previous month, and those who exercised $(61.5 \%)$ participated in $6.5 \pm 5.6 \mathrm{~h}$ of planned exercise per week. Among the 107 persons (61.5\%) who reported planned exercise, 47 persons reported time participating in stretching, range of motion, or physical therapy, accounting for $19.9 \%$ of all exercise time, and 93 persons reported time spent in other exercise activities $(80.1 \%$ of all exercise time). 
In analyses assessing individual factors associated with 25(OH)D (Table 2), there was a statistically significant positive association between $25(\mathrm{OH}) \mathrm{D}$ with total vitamin $\mathrm{D}$ intake (Fig. 1), age, years of education, total calcium intake, wine consumption, total hours of planned exercise, female sex, white race, and among never smokers, and users of sunscreen. Planned exercise reported as participating in stretching, range of motion, or physical therapy was not associated with $25(\mathrm{OH}) \mathrm{D}$, whereas other planned exercise was associated with $25(\mathrm{OH}) \mathrm{D}$. There was no significant relationship between $25(\mathrm{OH}) \mathrm{D}$ and SCI level and completeness of injury, BMI, \%total body fat, mobility mode, comorbid medical conditions, time outside between sunrise and sunset, or season.

In a multivariable model (Table 3 ), age, total vitamin D intake, total hours of planned exercise, sex, race, wine use, and smoking status remained statistically significantly associated with $25(\mathrm{OH}) \mathrm{D}$. Race and total vitamin D intake were the most statistically significant $(p<0.001)$ predictors. The regression coefficient for total vitamin $D$ intake (Table 3) remained significant but was reduced by approximately $21 \%$ in the final multivariable model compared to the univariable model (Table 2). A multivariable model with the effects of vitamin $\mathrm{D}$ intake divided into dietary intake and supplement use was assessed, adjusting for age, planned exercise, sex, race, wine use, and smoking status. When comparing the impacts of vitamin D from diet and from supplements, supplement intake was statistically significantly associated with increased $25(\mathrm{OH})$ D $(p<0.001)$, but dietary intake was not $(p=0.305)$ (Table 3 ). We also assessed stretching, range of motion, or physical therapy and other planned exercise as separate covariates. As in the univariable model, stretching, range of motion, or physical therapy was not associated with higher $25(\mathrm{OH}) \mathrm{D}$ level and other planned exercise was associated with higher $25(\mathrm{OH}) \mathrm{D}$ levels. In the

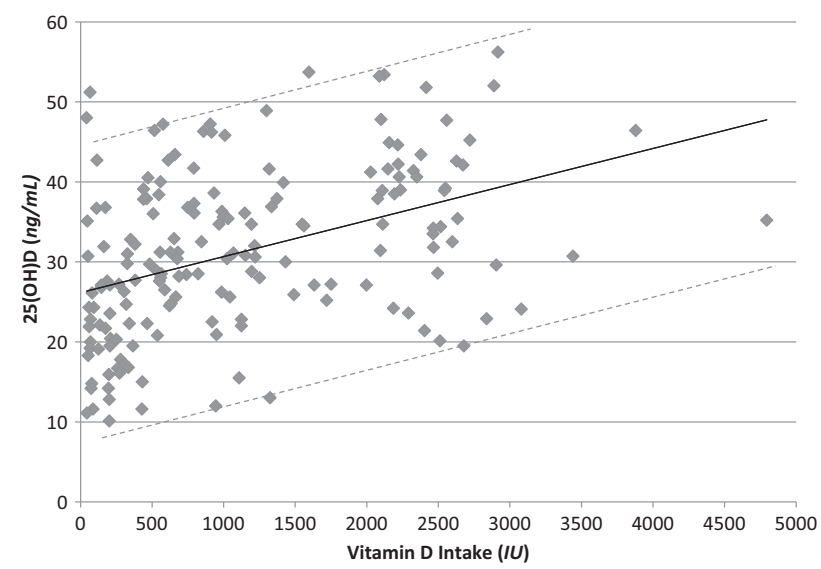

Fig. 1 Plasma 25(OH)D levels and daily vitamin D intake with the fit line and $95 \%$ confidence intervals multivariable model, several variables were no longer statistically significant, specifically sunscreen use, calcium intake from diet and supplements, and education level. These variables were excluded from the final model.

As noted, the FFQ was not completed during the study visit when $25(\mathrm{OH}) \mathrm{D}$ levels were assessed, so responses regarding vitamin D intake, including from multivitamins, obtained at each study visit were compared to the information provided on the food frequency questionnaire. There were 17 persons whose responses on vitamin D or multivitamin supplement usage on the FFQ were discordant with

Table 3 Final multivariable model

\begin{tabular}{|c|c|c|}
\hline Continuous variables & $\beta(95 \% \mathrm{CI})$ & $P$ \\
\hline Age (years) & $0.142(0.032,0.251)$ & 0.012 \\
\hline $\begin{array}{l}\text { Total daily vitamin D intake } \\
\text { (per } 100 \mathrm{IU} \text { ) }\end{array}$ & $0.355(0.219,0.490)$ & $<0.001$ \\
\hline Planned exercise (h/week) & $0.267(0.029,0.505)$ & 0.028 \\
\hline Categorical variables & Mean $(\mathrm{ng} / \mathrm{mL}) \pm \mathrm{SE}$ & $P$ \\
\hline Sex & & 0.011 \\
\hline Male & $28.6 \pm 1.3$ & \\
\hline Female & $32.9 \pm 1.8$ & \\
\hline Race & & $<0.001$ \\
\hline White & $34.3 \pm 1.1$ & \\
\hline Other & $27.2 \pm 2.0$ & \\
\hline Wine use (drinks per month) & & 0.030 \\
\hline Above median $(>12)$ & $33.0 \pm 2.1$ & $P$ trend 0.015 \\
\hline Below median $(\leq 12)$ & $30.9 \pm 1.9$ & \\
\hline None & $28.3 \pm 1.2$ & \\
\hline Smoking status & & 0.010 \\
\hline Current & $28.5 \pm 2.4$ & $P$ trend 0.003 \\
\hline Former & $29.9 \pm 1.3$ & \\
\hline Never & $33.8 \pm 1.5$ & \\
\hline $\begin{array}{l}\text { Vitamin D by diet and } \\
\text { supplement }^{\mathrm{a}}\end{array}$ & $\beta(95 \% \mathrm{CI})$ & $P$ \\
\hline $\begin{array}{l}\text { Vitamin D intake from diet } \\
\text { (per } 100 \mathrm{IU} \text { ) }\end{array}$ & $\begin{array}{l}0.471(-0.528 \\
1.470)\end{array}$ & 0.353 \\
\hline $\begin{array}{l}\text { Vitamin D intake from } \\
\text { supplements (per } 100 \mathrm{IU})\end{array}$ & $0.353(0.216,0.489)$ & $<0.001$ \\
\hline Planned exercise ${ }^{\mathrm{b}}$ & $\beta(95 \% \mathrm{CI})$ & $P$ \\
\hline $\begin{array}{l}\text { Stretching/range of motion/ } \\
\text { physical therapy (h/week) }\end{array}$ & $\begin{array}{l}0.097(-0.561 \\
0.755)\end{array}$ & 0.771 \\
\hline Other exercise (h/week) & $0.297(0.034,0.559)$ & 0.027 \\
\hline
\end{tabular}

${ }^{a}$ Adjusting for age, planned exercise, sex, race, wine use, and smoking status

${ }^{\mathrm{b}}$ Adjusting for age, total daily vitamin D intake, sex, race, wine use, and smoking status 
their responses at the study visit. We repeated the multivariable models excluding these persons and noted no difference in the results. Laboratory batch was also not significantly associated with $25(\mathrm{OH}) \mathrm{D}$ in the final model. There were 32 persons who completed a visit between the study dates but who did not return a FFQ. There was no significant difference in level and completeness of injury or in available mean $25(\mathrm{OH}) \mathrm{D}$ levels compared to the responders.

\section{Discussion}

In this cross-sectional analysis of persons with chronic SCI, SCI level and completeness of injury, mobility, and duration of injury were not found to be determinants of $25(\mathrm{OH})$ D. Instead, other factors based on personal, nutritional, and lifestyle characteristics were found to be statistically significant predictors of $25(\mathrm{OH}) \mathrm{D}$. Total vitamin D intake was found to be the most closely associated with plasma $25(\mathrm{OH})$ D levels.

Nutritional characteristics of the population appeared to be the most important determinant of vitamin D status. Higher levels of total vitamin D intake, which came from dietary and supplemental sources, were closely associated with higher levels of plasma 25(OH)D. A number of studies have assessed daily vitamin D intake in chronic SCI, in which they found intake levels to be inadequate [16-18, 30]. Like these studies, we also found that the median vitamin D dietary intake for our population was below the IOM recommendation. Even though diet provides an important source of vitamin $\mathrm{D}$, based on our population's dietary habits, supplemental usage was a more effective determinant of plasma 25(OH)D status than dietary vitamin D. Participants who were vitamin D sufficient were consuming considerably more supplemental vitamin $\mathrm{D}$ than participants who were vitamin $\mathrm{D}$ deficient. This is in contrast to persons across all levels of vitamin D sufficiency consuming similar amounts of vitamin D from their diet. As with vitamin D intake, calcium dietary intake also did not meet national recommendations for most individuals and supplements were necessary for the population to attain the recommended intake levels of calcium.

Certain lifestyle habits such as tobacco use and exercise had an association with 25(OH)D levels. Exercise has been shown to have a number of health benefits in SCI [31-33]. In our study, planned exercise also had a positive association with plasma $25(\mathrm{OH}) \mathrm{D}$ that appeared attributable to exercise other than physical therapy, stretching, and range of motion exercises that are applicable to patients with SCI. In addition to exercise, smoking status was negatively associated with $25(\mathrm{OH}) \mathrm{D}$, with the lowest levels in current smokers. Although the mechanism is uncertain, previous studies have similarly shown the negative effects of smoking on $25(\mathrm{OH}) \mathrm{D}$ levels in individuals without SCI $[9,10,34]$.

In addition to diet and smoking status, the effects of wine drinking were also significantly associated with circulating 25(OH)D levels. Although wine contains no vitamin D, wine consumption displayed a positive association with plasma $25(\mathrm{OH}) \mathrm{D}$ specifically between non-drinkers and drinkers consuming greater than 12 drinks per month. A Swiss study in 2012 similarly reported greater levels of 25 $(\mathrm{OH}) \mathrm{D}$ amongst heavy wine drinkers [12]. It is unclear how wine may influence $25(\mathrm{OH}) \mathrm{D}$ levels, though it is suggested that certain phytoestrogens found in red wine may enhance the levels of 25(OH)D [35].

Personal characteristics such as age, sex, and race were also found to be closely associated with plasma $25(\mathrm{OH}) \mathrm{D}$ levels. It is unclear how greater age or female sex may be positively associated with vitamin D status, but it is believed that race performed as a proxy for skin tone in this study, which influences the amount of sunlight that is absorbed into the skin and catalyzes vitamin $\mathrm{D}_{3}$ synthesis [1]. Previous studies have shown that persons with fairer skin complexions generally have higher levels of plasma 25 (OH)D [36, 37].

We found no association with BMI or \%total body fat even though lesser levels of plasma 25(OH)D have been associated with a greater BMI and total body fat in some studies $[15,18]$. It is possible that with a larger sample size we may have observed an association since participants who were obese had slightly lower levels of $25(\mathrm{OH}) \mathrm{D}$. Although direct sun exposure was not measured in our study, the use of sunscreen was positively associated with 25(OH)D levels in the univariable model, possibly as a marker of greater sun exposure. Sunscreen use was no longer significant, however, in the multivariable model. Additionally, while our population reported spending an average of almost $2 \mathrm{~h}$ per day outside between sunrise and sunset, time outside had no association with plasma $25(\mathrm{OH}) \mathrm{D}$ status. These results may be reflective of the participants all living in the New England area where direct sun exposure does not occur during the winter. We examined time outside between sunrise and sunset in only the months of April through September to concentrate on relevant sun exposure, but still found no significant association with $25(\mathrm{OH}) \mathrm{D}$ level. Moreover, there was no association found in $25(\mathrm{OH}) \mathrm{D}$ levels with season.

A strength of this study is the use of a validated food frequency questionnaire to assess the vitamin D and calcium intake. Moreover, our comprehensive assessment of participants provided us with a wide range of detailed information on multiple SCI related and 
personal factors that were used to find associations with 25(OH)D.

It is possible that questions in our health questionnaire could have better assessed direct sun exposure instead of time spent outside between sunrise and sunset. A welldefined assessment of direct sunlight exposure may have shown an association between higher levels of $25(\mathrm{OH}) \mathrm{D}$ and greater sun exposure like other studies have suggested. However, associations between measures of sunlight exposure and plasma 25(OH)D have not been consistently noted as described in a report that assessed ultraviolet radiation exposure based on residential address in three large non-SCI large cohort studies [38]. Additionally, completion of the FFQ on the same date as the study visit or a detailed personal interview may have more accurately represented dietary intake at the time when the blood draw was collected and therefore could have increased the accuracy of the associations between plasma 25(OH)D and the vitamin $\mathrm{D}$ and calcium intake variables. Finally, subjects in the study had to be healthy enough to travel to the VA, which could have potentially limited our ability to study persons with greater levels of injury and completeness of SCI.

To summarize, plasma $25(\mathrm{OH}) \mathrm{D}$ levels were significantly associated with dietary intake in this chronic SCI cohort and were unrelated to injury level and completeness of SCI. Usual dietary intake was considerably less than the recommended daily intake and supplement intake was most closely associated with adequate levels. Other factors related to lifestyle including planned exercise, wine consumption, and smoking status were also determinants of $25(\mathrm{OH}) \mathrm{D}$ status. Our study suggests that clinicians may want to consider recommending vitamin D supplement use in SCI patients and to provide information to patients on personal lifestyle choices that may impact 25(OH)D levels.

Acknowledgements We thank Samuel Davis, Boston VA Healthcare System, technician, for DXA data collection.

Funding This work was supported by Merit Review B6618R and I01 RX000792 from the United States Department of Veterans Affairs Rehabilitation Research and Development Service Program and National Institutes of Health National Institute of Arthritis and Musculoskeletal and Skin Diseases R01 AR059270. The views expressed in this article are those of the authors and do not necessarily reflect the position or policy of the Department of Veterans Affairs or the United States Government.

Author contributions EG, AAL, CGT contributed to study conception and design. EG, NEK, RLG, PW, and MMP contributed to data analysis. The draft manuscript was prepared by NEK, EG, RLG, and JEH. NEK, EG, RLG, and JEH edited and revised the manuscript. All authors read and approved the final manuscript.

\section{Compliance with ethical standards}

Conflict of interest The authors declare that they have no conflict of interest.

\section{References}

1. Holick MF. Vitamin D deficiency. N Engl J Med. 2007;357:266-81.

2. Willhelm-Leen ER, Hall YN, deBoer IH, Chertow GM. Vitamin $\mathrm{D}$ deficiency and frailty in older Americans. J Int Med. 2010;268:171-80.

3. Ceglia L. Vitamin D and its role in skeletal muscle. Curr Opin Clin Nutr Metab Care. 2009;12:628-33.

4. Black PN, Scragg R. Relationship between serum 25hydroxyvitamin $\mathrm{D}$ and pulmonary function in the third national health and nutrition examination survey. Chest. 2005;128: 3792-8.

5. Janssens W, Bouillon R, Claes B, Carremans C, Lehouck A, Buysschaert I, et al. Vitamin D deficiency is highly prevalent in COPD and correlates with variants in the vitamin D-binding gene. Thorax. 2010;65:215-20.

6. Manson JE. Vitamin D and the heart: why we need large-scale clinical trials. Clevel Clin J Med. 2010;77:903-10.

7. Hummel K, Craven BC. Serum 25(OH)D, PTH and correlates of suboptimal $25(\mathrm{OH}) \mathrm{D}$ levels in persons with chronic spinal cord injury. Spinal Cord. 2012;50:812-6.

8. Touvier M, Deschasaux M, Montourcy M, Sutton A, Charnaux N, Kesse-Guyot E, et al. Determinants of vitamin D status. J Inv Dermatol. 2015;135:378-88.

9. Tønnesen R, Hovind PH, Jensen LT, Schwarz P. Determinants of vitamin D status in young adults: influence of lifestyle, sociodemographic and anthropometric factors. BMC Public Health. 2016;385:1-11.

10. Kühn T, Kaaks R, Teucher B, Hirche F, Dierkes J, Weikert C, et al. Dietary, lifestyle, and genetic determinants of vitamin D status: a cross-sectional analysis from the European prospective investigation into cancer and nutrition (EPIC)-Germany study. Eur J Nutr. 2014;53:731-41.

11. George JA, Norris SA, van Deventer HE, Pettifor JM, Crowther NJ. Effect of adiposity, season, diet and calcium or vitamin D supplementation on vitamin D status of healthy urban African and Asian-Indian adults. Br J Nutr. 2014;112:590-9.

12. Guessous I, Dudler V, Glatz N, Theler JM, Zoller O, Paccaud F, et al. Vitamin D levels and associated factors: a population-based study in Switzerland. Swiss Med Wkly. 2012;142:w13719.

13. Spiro A, Buttriss JL. Vitamin D: an overview of vitamin D status and intake in Europe. Nutr Bull. 2014;39:322-50.

14. Lamarche J, Mailhot G. Vitamin D and spinal cord injury: should we care? Spinal Cord. 2016;54:1060-75.

15. Flueck JL, Perret C. Vitamin D deficiency in individuals with a spinal cord injury: a literature review. Spinal Cord. 2017;55:428-34.

16. Pritchett K, Pritchett R, Ogan D, Bishop P, Broad E, LaCroix M. $25(\mathrm{OH}) \mathrm{D}$ status of elite athletes with spinal cord injury relative to lifestyle factors. Nutrients. 2016;8:E374.

17. Perret C, Stoffel-Kurt N. Comparison of nutritional intake between individuals with acute an chronic spinal cord injury. J Spinal Cord Med. 2011;34:569-75.

18. Walters JL, Buchholz AC, Martin Ginis KA, SHAPE-SCI Research Group. Evidence of dietary inadequacy in adults with chronic spinal cord injury. Spinal Cord. 2009;47:318-22. 
19. Millen AE, Bodnar LM. Vitamin D assessment in populationbased studies: a review of the issues. Am J Clin Nutr. 2008;87 (suppl):1102S-5S.

20. Willett WC, Sampson L, Stampfer MJ, Rosner B, Bain C, Witschi $\mathrm{J}$, et al. Reproducibility and validity of a semiquantitative food frequency questionnaire. Am J Epidemiol. 1985;122:51-65.

21. Willett WC, Sampson L, Browe ML, Stampfer MJ, Rosner B, Hennekens C, et al. The use of a self-administered questionnaire to assess diet four year in the past. Am J Epidemiol. 1988;127: 188-99.

22. Rimm EB, Giovannucci E, Stampfer MJ, Colditz GA, Litin L, Willett W. Reproducibility and validity of a self-administered semi-quantitative food frequency questionnaire among male health professionals. Am J Epidemiol. 1992;135:418-27.

23. Ferris BG. Epidemiology standardization project (American Thoracic Society). Am Rev Respir Dis. 1978;188:1-120.

24. Wang H, Chen W, Li D, Yin X, Zhang X, Olsen N, et al. Vitamin D and chronic diseases. Aging Dis. 2017;8:346-53.

25. McCarty CA. Sunlight exposure assessment: can we accurately assess vitamin D exposure form sunlight questionnaires? Am J Clin Nutr. 2008;87:1907S-2101S.

26. Kirshblum SC, Waring W, Biering-Sorensen F, Burns SP, Johansen M, Schmidt-Read M, et al. Reference for the 2011 revision of the international standards for neurological classifications of spinal cord injury. J Spinal Cord Med. 2011;34:547-54.

27. DeVivo MJ, Biering-Sørensen F, New P, Chen Y. Standardization of data analysis and reporting of results from the international spinal cord injury core data set. Spinal Cord. 2011;49:596-9.

28. Holick MF, Binkley NC, Bischoff-Ferrari HA, Gordon CM, Hanley DA, Heaney RP, et al. Evaluation, treatment, and prevention of vitamin D deficiency: an endocrine society clinical practice guideline. J Clin Endocrinol Metab. 2011;96:1911-30.

29. Institute of Medicine, Food and Nutrition Board. Dietary reference intakes for calcium and vitamin D. The National Academies Press: Washington D.C.; 2011.
30. Javidan AN, Sabour H, Latifi S, Vafa M, Shidfar F, Khazaeipour $\mathrm{Z}$, et al. Calcium and vitamin D plasma concentration and nutritional intake status in patients with chronic spinal cord injury: a referral center report. J Red Med Sci. 2014;19: $881-4$.

31. Garshick E, Mulroy S, Graves DE, Greenwald K, Horton JA, Morse LR. Active lifestyle is associated with reduced dyspnea and greater life satisfaction in spinal cord injury. Arch Phys Med Rehabil. 2016;97:1721-7.

32. Neefkes-Zonneveld CR, Bakkum AJ, Bishop NC, van Tulder MW, Janssen TW. Effect of long-term physical activity and acute exercise on markers of systematic inflammation in persons with chronic spinal cord injury: a systematic review. Arch Phys Med Rehabil. 2016;96:30-42.

33. Yamamoto M, Tajima F, Okawa H, Mizushima T, Umezu Y, Ogata H. Static exercise-induced increase in blood pressure in individuals with cervical spinal cord injury. Arch Phys Med Rehabil. 1999;80:288-93.

34. Brot C, Jorgensen NR, Sorensen $\mathrm{OH}$. The influence of smoking on vitamin D status and calcium metabolism. Eur J Clin Nutr. 1999;53:920-6.

35. Wietzke JA. Phytoestrogen regulation of vitamin D3 receptor promoter and 1,25-dihydroxyvitamin D3 actions in human breast cancer cells. J Steroid Biochem Mol Biol. 2003;84: 149-57.

36. Bonilla C, Ness AR, Wills AK, Lawlor DA, Lewis SJ, Davey Smith G. Skin pigmentation, sun exposure and vitamin D levels in children of the Avon longitudinal study of parents and children. BMC Public Health. 2014;14:597.

37. Harris SS. Vitamin D and African Americans. J Nutr. 2006;136:1126-9.

38. Bertrand KA, Giovannucci E, Liu Y, Malspeis S, Eliassen AH, $\mathrm{Wu} \mathrm{K}$, et al. Determinants of plasma 25-hydroxyvitamin D and development of prediction models in three US cohorts. Br J Nutr. 2012;108:1889-96. 